\title{
Amygdalin blocks the in vitro adhesion and invasion of renal cell carcinoma cells by an integrin-dependent mechanism
}

\author{
EVA JUENGEL ${ }^{1}$, MASUD AFSCHAR ${ }^{1}$, JASMINA MAKAREVIĆ ${ }^{1}$, JOCHEN RUTZ $^{1}$, IGOR TSAUR ${ }^{1}$, \\ JENS MANI $^{1}$, KAREN NELSON $^{2}$, AXEL HAFERKAMP $^{1^{*}}$ and ROMAN A. BLAHETA ${ }^{1 *}$ \\ Departments of ${ }^{1}$ Urology and ${ }^{2}$ Vascular and Endovascular Surgery, \\ Goethe-University Hospital, Frankfurt am Main, Germany
}

Received July 29, 2015; Accepted December 3, 2015

DOI: $10.3892 / \mathrm{ijmm} .2016 .2454$

\begin{abstract}
Information about the natural compound amygdalin, which is employed as an antitumor agent, is sparse and thus its efficacy remains controversial. In this study, to determine whether amygdalin exerts antitumor effects on renal cell carcinoma (RCC) cells, its impact on RCC metastatic activity was investigated. The RCC cell lines, Caki-1, KTC-26 and A498, were exposed to amygdalin from apricot kernels, and adhesion to human vascular endothelium, immobilized collagen or fibronectin was investigated. The influence of amygdalin on chemotactic and invasive activity was also determined, as was the influence of amygdalin on surface and total cellular $\alpha$ and $\beta$ integrin expression, which are involved in metastasis. We noted that amygdalin caused significant reductions in chemotactic activity, invasion and adhesion to endothelium, collagen and fibronectin. Using FACScan analysis, we noted that amygdalin also induced reductions, particularly in integrins $\alpha 5$ and $\alpha 6$, in all three cell lines. Functional blocking of $\alpha 5$ resulted in significantly diminished adhesion of KTC-26 and A498 to collagen and also in decreased chemotactic behavior in all three cell lines. Blocking $\alpha 6$ integrin significantly reduced chemotactic activity in all three cell lines. Thus, we suggest that exposing RCC cells to amygdalin inhibits metastatic spread and is associated with downregulation of $\alpha 5$ and $\alpha 6$ integrins. Therefore, we posit that amygdalin exerts antitumor activity in vitro, and this may be linked to integrin regulation.
\end{abstract}

\section{Introduction}

Renal cell carcinoma (RCC) is the most common renal tumor. Approximately one-third of patients have metastases

Correspondence to: Dr Eva Juengel, Department of Urology, Goethe-University Hospital, Interdisciplinary Science Building, Theodor-Stern-Kai 7, D-60590 Frankfurt am Main, Germany

E-mail: eva.juengel@kgu.de

*Contributed equally

Key words: amygdalin, cell adhesion, integrins, neoplasm invasion, renal cell carcinoma at diagnosis, and up to $30 \%$ of patients develop metastases during therapy. Once metastasized, the prognosis for patients is bleak. A better understanding of the molecular modes of action underlying RCC development and progression has contributed to the development of targeted therapies, thus improving the outlook for patients in the advanced stages of this disease. However, despite these therapeutic advances, the prognosis for patients with RCC remains poor, with 5-year survival remaining between 5 and $12 \%$. Dissatisfaction with conventional therapy and the desire to reduce side-effects have led many patients to complementary and alternative medicine (CAM). Up to $80 \%$ of cancer patients in the United States, and more than $50 \%$ of cancer patients in Europe use CAM alongside, or in place of, conventional therapy.

Information about the efficacy of natural compounds is sparse, and certain of these compounds, such as the cyanogenic diglycoside amygdalin (D-mandelonitrile- $\beta$-gentiobioside), remain controversial. Amygdalin is derived from the fruit kernels of the Rosaceae family, which includes Prunus persica (peach), Prunus armeniaca (apricot) and Prunus amygdalus var. amara (bitter almond). Amygdalin, mainly in the United States, has been administered to cancer patients since the 1920s. In the 1950s, an intravenous, chemically different form of amygdalin was synthesized and patented as laetrile. Although laetrile differs from amygdalin, the terms are often used interchangeably, making data interpretation difficult. By 1978 approximately 70,000 cancer patients in the US had been treated with amygdalin. Evidence-based research on amygdalin, however, remains limited. A clinical study sponsored by the National Cancer Institute over 30 years ago revealed no signs of tumor regression (1), whereas a retrospective analysis of 67 tumor patients receiving amygdalin reported two complete and four partial responses (2). Ambivalence is also reflected in case reports: amygdalin was ineffective in five cases, and effective in four. Randomized clinical trials and follow-up studies have not been carried out, to the best of our knowledge. Proponents consider amygdalin an effective natural cancer treatment option, whereas opponents warn of toxicity due to hydrogen cyanide metabolization.

Metastasis is the main cause of RCC-associated mortality. Transendothelial migration and motile spread are critical steps in tumor dissemination and progression (3), and the dissemination of cancer cells to distant organs constitutes the major 
clinical challenge in treating cancer. In the present study, the anti-neoplastic effect of amygdalin on RCC cell adhesion and migration properties was investigated. Since integrins activate a number of intracellular signaling pathways involved in cell proliferation, differentiation and motility, the expression pattern of $\alpha$ and $\beta$ integrin adhesion receptors between amygdalin-treated cells and untreated controls was determined. Integrins are important in both health and disease (4) and play a pivotal role in carcinogenesis and cancer progression (4).

The present study is based on a previous investigation dealing with the influence of amygdalin on the metastatic properties of three bladder cancer cell lines (5). Since some dissimilarities were observed regarding the action of amygdalin on the metastatic properties of the different bladder cancer cell lines, the question arose as to whether the different effects of amygdalin were restricted to particular tumor entities or occur in others. Thus, three RCC cell lines were chosen, since RCC tumors are the most aggressive urologic tumor.

\section{Materials and methods}

Cell culture. Kidney carcinoma cells, Caki-1, KTC-26, and A498, were purchased from LGC Promochem GmbH (Wesel, Germany). The cells were grown and subcultured in RPMI-1640 medium (Seromed, Berlin, Germany) supplemented with $10 \%$ fetal calf serum (FCS), $20 \mathrm{mM}$ HEPES buffer, $100 \mathrm{IU} / \mathrm{ml}$ penicillin and $100 \mu \mathrm{g} / \mathrm{ml}$ streptomycin at $37^{\circ} \mathrm{C}$ in a humidified incubator with $5 \% \mathrm{CO}_{2}$. Subcultures from passages 5-24 were selected for experimental use. Human umbilical vein endothelial cells (HUVECs) were isolated from human umbilical veins and harvested by enzymatic treatment with dispase $(1 \mathrm{U} / \mathrm{ml}$; Gibco-Invitrogen, Carlsbad, CA, USA). HUVECs were grown in Medium 199 (M199; Biozol, Munich, Germany), supplemented with $10 \%$ FCS, $10 \%$ pooled human serum, $20 \mu \mathrm{g} / \mathrm{ml}$ endothelial cell growth factor (Boehringer, Mannheim, Germany), $0.1 \%$ heparin, $100 \mathrm{ng} / \mathrm{ml}$ gentamycin and $20 \mathrm{mM}$ HEPES buffer ( $\mathrm{pH}$ 7.4). Subcultures from passages 1-5 were selected for experimental use. The Institutional Ethics Committee of Goethe-University Hospital, Frankfurt, Germany, waived the need for consent since HUVECs were used anonymously for in vitro assays and had no links with patient data.

Amygdalin treatment. Amygdalin from apricot kernels (Sigma-Aldrich, Taufkirchen, Germany) was freshly dissolved in cell culture medium and then added to tumor cells at a concentration of $10 \mathrm{mg} / \mathrm{ml}$ [previously evaluated as optimal concentration (6)] for either $24 \mathrm{~h}$ or for 2 weeks (treatment applied three times a week) to evaluate acute versus chronic treatment. Controls remained untreated. In all experiments, treated and non-treated tumor cell cultures were compared. To examine the toxic effects of amygdalin, cell viability was determined by trypan blue (Gibco-Invitrogen).

Tumor cell adhesion. To analyze tumor cell adhesion, HUVECs were transferred to 6-well multiplates (Sarstedt, Nümbrecht, Germany) in complete HUVEC medium. When they reached confluence, Caki-1, KTC-26 and A498 cells were detached from the culture flasks by treatment with accutase (PAA Laboratories, Cölbe, Germany), and $0.5 \times 10^{6}$ cells were then added to and left on the HUVEC monolayer for $1,2 \mathrm{or} 4 \mathrm{~h}$. Subsequently, non-adherent tumor cells were washed off using warmed $\left(37^{\circ} \mathrm{C}\right)$ PBS $\left(\mathrm{Ca}^{2+}\right.$ and $\left.\mathrm{Mg}^{2+}\right)$. The remaining cells were fixed with $1 \%$ glutaraldehyde. Adherent tumor cells were counted in five different fields of a defined size $\left(5 \times 0.25 \mathrm{~mm}^{2}\right)$ using a phase contrast microscope (ID03, 471202-9903; Carl Zeiss Microscopy $\mathrm{GmbH}$, Goettingen, Germany), and the mean cellular adhesion rate was calculated.

Attachment to immobilized extracellular matrix proteins. The 24-well plates were coated with collagen $\mathrm{G}$ (extracted from calfskin, consisting of $90 \%$ collagen type I and $10 \%$ collagen type III, and diluted to $400 \mu \mathrm{g} / \mathrm{ml}$ in PBS; Biochrom, Berlin, Germany) or fibronectin (extracted from mice and diluted to $100 \mu \mathrm{g} / \mathrm{ml}$ in PBS; Becton-Dickinson, Heidelberg, Germany) overnight. Plastic dishes served as the background control. Plates were washed with $1 \%$ bovine serum albumin (BSA) in PBS to block non-specific cell adhesion. Tumor cells $\left(0.1 \times 10^{6}\right)$ were then added to each well and left for 30 min incubation. Subsequently, non-adherent tumor cells were washed off, the remaining adherent cells were fixed with $2 \%$ glutaraldehyde and counted microscopically. The mean cellular adhesion rate, defined by adherent cells $s_{\text {coated well }}$ - adherent cells background $_{\text {, was }}$ calculated from five different observation fields.

Chemotactic activity. Serum-induced chemotactic movement was examined using 6-well Transwell chambers (Greiner, Frickenhausen, Germany) with $8-\mu \mathrm{m}$ pores. The cells $\left(0.5 \times 10^{6}\right.$ Caki-1, KTC-26 or A498 cells $/ \mathrm{ml}$ ) were placed in the upper chamber in serum-free medium, either free of amygdalin (control) or containing amygdalin. The lower chamber contained 10\% serum. Following overnight incubation, the upper surface of the transwell membrane was gently wiped with a cotton swab to remove cells that had not migrated. Cells moving to the lower surface of the membrane were stained using hematoxylin and counted microscopically. The mean migration rate was calculated from five different observation fields.

Invasion. Invasion was examined by serum-induced chemotactic movement through a membrane (Greiner) with $8-\mu \mathrm{m}$ pores, pre-coated with collagen $\mathrm{G}$ (extracted from calfskin, consisting of $90 \%$ collagen type I and $10 \%$ collagen type III; diluted to $400 \mu \mathrm{g} / \mathrm{ml}$ in PBS; Biochrom) and HUVECs, grown to confluence. Caki-1, KTC-26 or A498 cells $\left(0.5 \times 10^{6} / \mathrm{ml}\right)$ were placed in the upper chamber in serum-free medium, either free of amygdalin (control) or containing amygdalin. The lower chamber contained $10 \%$ serum. After overnight incubation, the upper surface of the transwell membrane was gently wiped with a cotton swab to remove cells which had not migrated. Cells which had moved to the lower surface of the membrane were stained using hematoxylin and counted microscopically. The mean migration rate was calculated in five different observation fields.

Integrin surface expression. Tumor cells were washed in blocking solution (PBS, $0.5 \% \mathrm{BSA}$ ) and then incubated for $60 \mathrm{~min}$ at $4^{\circ} \mathrm{C}$ with phycoerythrin (PE)-conjugated monoclonal antibodies directed against the following integrin subtypes: anti- $\alpha 1$ (mouse IgG1; clone SR84; \#559596), anti- $\alpha 2$ (mouse IgG2a; clone 12F1- 
H6; \#555669), anti- $\alpha 3$ (mouse IgG1; clone C3II.1; \#556025), anti- $\alpha 4$ (mouse IgG1; clone 9F10; \#555503), anti- $\alpha 5$ (mouse IgG1; clone IIA1; \#555617), anti- $\alpha 6$ (mouse IgG2a; clone GoH3; \#555736), anti- $\beta 1$ (mouse IgG1; clone MAR4; \#555443), anti- $\beta 3$ (mouse IgG1; clone VI-PL2; \#555754) or anti- $\beta 4$ (rat IgG2b; clone 439-9B; \#555720) (all from BD Pharmingen, Heidelberg, Germany). Integrin expression of tumor cells was then measured using a FACScan (BD Biosciences, Heidelberg; FL-2H (log) channel histogram analysis; $1 \times 10^{4}$ cells/scan) and expressed as mean relative fluorescence intensity (RFI). Mouse IgG1-PE (MOPC-21; \#555749), IgG2a-PE (G155-178; \#555574) and rat IgG2b-PE (R35-38; \#555848; all from BD Biosciences) were used as isotype controls.

Western blotting. To investigate integrin content, tumor cell lysates were applied to a 7-12\% polyacrylamide gel (depending on protein size) and electrophoresed for $90 \mathrm{~min}$ at $100 \mathrm{~V}$. The protein was then transferred to nitrocellulose membranes. After blocking with non-fat dry milk for $1 \mathrm{~h}$, the membranes were incubated overnight with the following antibodies: integrin $\alpha 1$ (rabbit, polyclonal, 1:1,000; \#AB1934; Chemicon/Millipore $\mathrm{GmbH}$, Schwalbach, Germany), integrin $\alpha 2$ (mouse IgG1, 1:250, clone 2; \#611017; BD Biosciences), integrin $\alpha 3$ (rabbit, polyclonal, 1:1,000; \#AB1920; Chemicon/Millipore $\mathrm{GmbH}$ ), integrin $\alpha 4$ (mouse, 1:200, clone: C-20; \#sc-6589; Santa Cruz Biotechnology, Inc., Santa Cruz, CA, USA)], integrin $\alpha 5$ (mouse IgG2a, 1:5,000, clone 1; \#610634; BD Biosciences), integrin $\alpha 6$ (rabbit, 1:200, clone H-87; \#sc-10730; Santa Cruz Biotechnology, Inc.), and integrin $\beta 1$ (mouse IgG1, 1:2,500, clone 18; \#610468), integrin $\beta 3$ (mouse IgG1, 1:2,500, clone 1; \#611141) and integrin $\beta 4$ (mouse IgG1, 1:250, clone 7; \#611233) (all from BD Biosciences). HRP-conjugated goat anti-mouse IgG and HRP-conjugated goat anti-rabbit IgG (both 1:5,000; Upstate Biotechnology, Lake Placid, NY, USA) served as the secondary antibodies. Additionally, integrin-related signaling was explored by anti-integrin-linked kinase (ILK) (clone 3, dilution 1:1,000; \#611803), anti-focal adhesion kinase (FAK) (clone 77, dilution 1:1,000; \#610088) and anti-p-specific FAK (pY397; clone 18, dilution 1:1,000; \#611807) antibodies (all from BD Biosciences). HRP-conjugated goat-anti-mouse IgG (dilution 1:5,000; Upstate Biotechnology) served as secondary antibodies. The membranes were briefly incubated with ECL detection reagent $\left(\mathrm{ECL}^{\mathrm{TM}}\right.$; Amersham, GE Healthcare, München, Germany) to visualize the proteins and then analyzed with the Fusion FX7 system (Peqlab, Erlangen, Germany). $\beta$-actin (1:1,000; Sigma-Aldrich) served as the internal control.

Gimp 2.8 software was used to perform pixel density analysis of the protein bands. The ratio of protein intensity $/ \beta$-actin intensity was calculated, and expressed as a percentage, related to controls set to $100 \%$.

Blocking experiments. To determine whether integrin $\alpha 5$ and $\alpha 6$ impacted the metastatic spread independently of amygdalin in Caki-1, KTC-26, and A498 cell lines, cells were incubated for $60 \mathrm{~min}$ with $10 \mu \mathrm{g} / \mathrm{ml}$ function-blocking anti-integrin $\alpha 5$ (clone P1D6) mouse mAb or anti-integrin $\alpha 6$ (clone NKI-GoH3) rat mAb (both from Millipore). Controls were incubated with cell culture medium alone. Subsequently, tumor cell adhesion to immobilized collagen as well as chemotaxis were analyzed as described above.
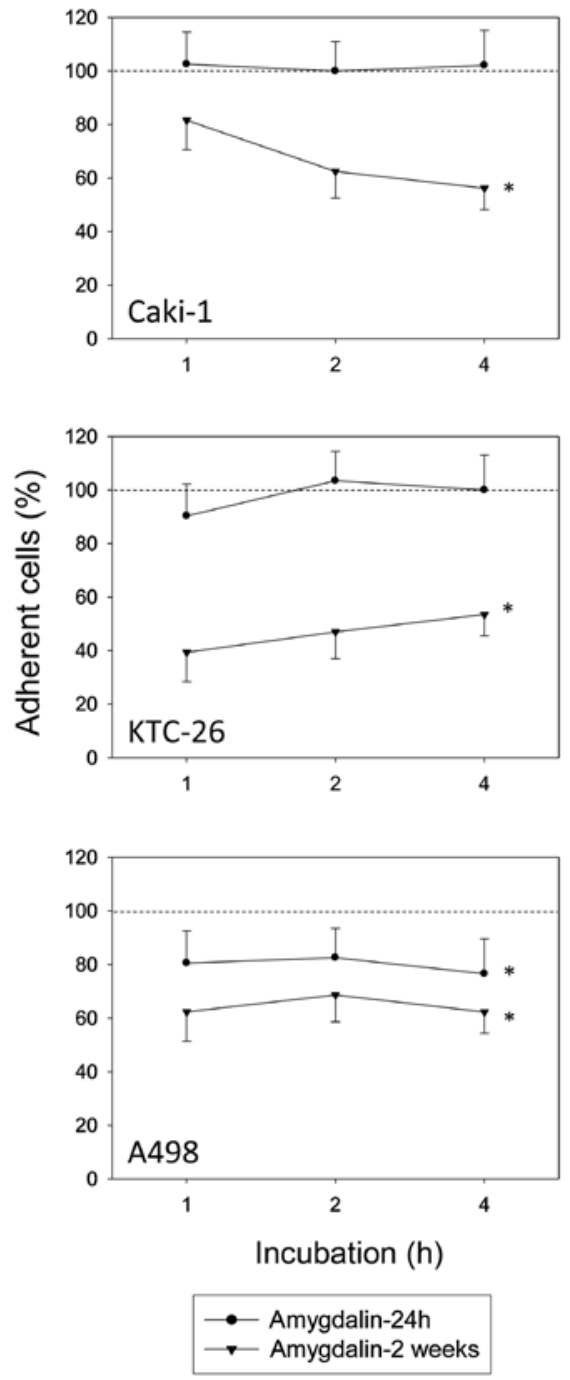

Figure 1. Adhesion of Caki-1, KTC-26 and A498 to endothelium (human umbilical endothelial cells; HUVECs). Tumor cells were treated with $10 \mathrm{mg} / \mathrm{ml}$ amygdalin for $24 \mathrm{~h}$ or 2 weeks. Controls remained untreated. Tumor cells $\left(0.5 \times 10^{6}\right.$ cells/well) were added to and left on the HUVEC monolayer for 1, 2 or $4 \mathrm{~h}$. The mean number of adherent tumor cells from five fields was evaluated, and the percentage of treated renal cell carcinoma (RCC) cells compared to untreated control cells (set to $100 \%$, dotted line) was calculated. *Significant difference to controls. Bars indicate the means \pm standard deviation (SD). $\mathrm{n}=5$ experiments, $\mathrm{p} \leq 0.05$.

Statistical analysis. In the present study, all experiments were performed 3-6 times. Statistical significance was determined using the Wilcoxon, Mann-Whitney U-test. A p-value $<0.05$ was considered to indicate a statistically significant difference.

\section{Results}

Amygdalin blocks interaction between the tumor cell endothelium and tumor cell matrix. After $24 \mathrm{~h}$ of treatment with amygdalin, A498 tumor cell adhesion to HUVECs was significantly diminished, but cell adhesion of Caki-1 and KTC-26 cells was not (compared to untreated controls set to $100 \%$ ) (Fig. 1). Extending the exposure time of amygdalin to 2 weeks significantly decreased adhesion to HUVECs in all three tumor cell lines (Fig. 1).

Amygdalin caused a significant decrease in the binding capacity of all three RCC cell lines to immobilized collagen 
A
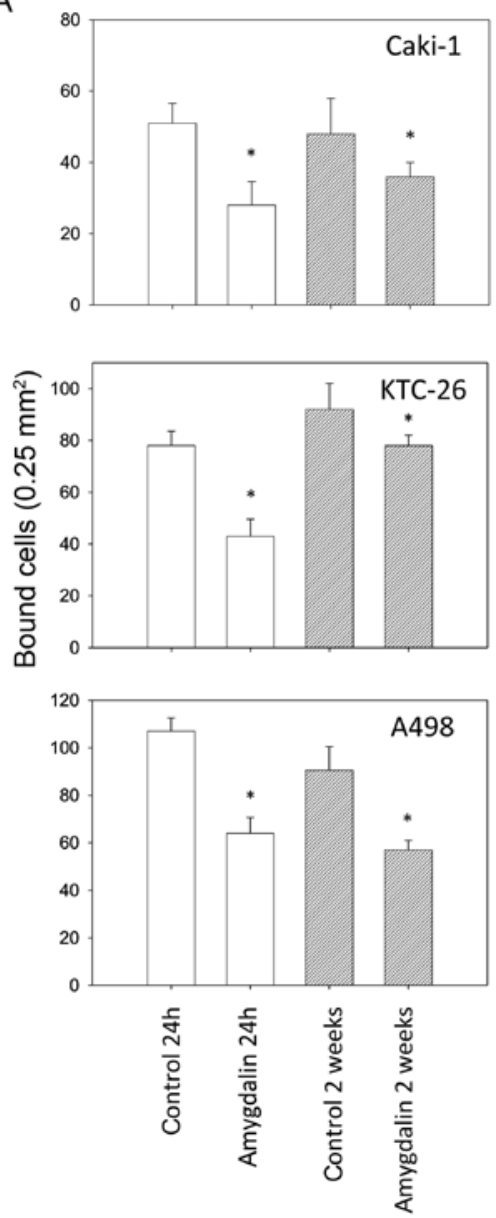

B
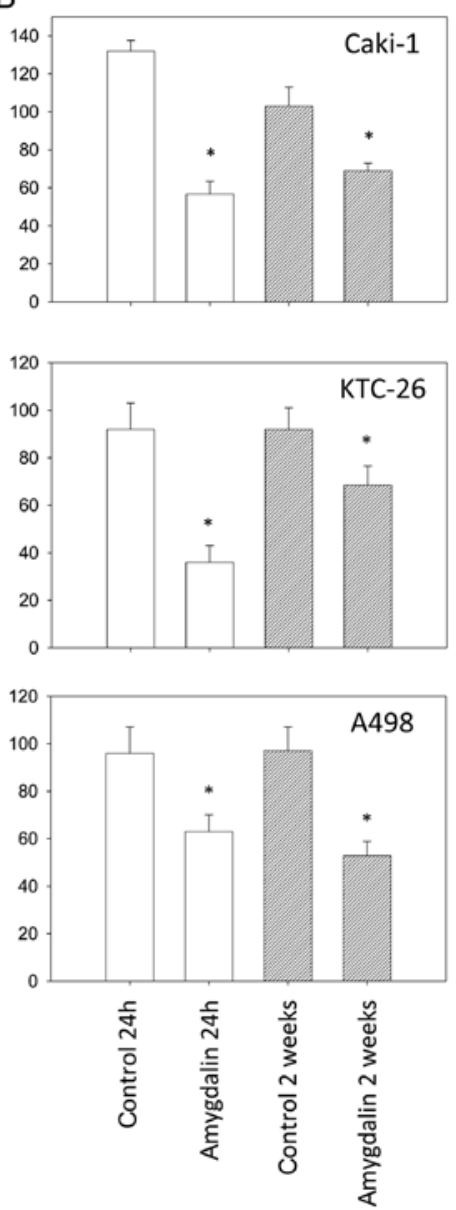

Figure 2. (A) Adhesion of Caki-1, KTC-26 and A498 to immobilized collagen (left) and (B) fibronectin (right). Tumor cells were treated with 10 mg/ml amygdalin for either $24 \mathrm{~h}$ or 2 weeks. Untreated renal cell carcinoma (RCC) cells served as controls. Cells $\left(0.1 \times 10^{6}\right.$ cells/well) were added to immobilized collagen or fibronectin. The mean number of adherent tumor cells from five fields was calculated after 30 min. *Significant difference to controls. Bars indicate the means \pm standard deviation (SD). $\mathrm{n}=6$ experiments, $\mathrm{p} \leq 0.05$.

and fibronectin, compared to controls (Fig. 2). Attachment of all three cell lines to the matrix proteins was diminished after $24 \mathrm{~h}$, as well as after 2 weeks. In the KTC-26 cell line, short-term amygdalin application (24 h) induced a greater decrease in adhesion than long-term amygdalin application (2 weeks).

Amygdalin alters the tumor cell motility of RCC cells. The chemotactic activity of Caki-1, KTC-26 and A498 cells significantly decreased after $24 \mathrm{~h}$ and 2 weeks of amygdalin application, compared to the untreated control cells (Fig. 3A). Tumor cell invasion through the collagen-coated transwell membranes was also significantly diminished in Caki-1 and A498 cells after $24 \mathrm{~h}$ and 2 weeks of amygdalin application (Fig. 3B). However, 2 weeks of amygdalin did not reduce the invasive capacity of KTC-26 cells.

Amygdalin modulates integrin $\alpha$ and $\beta$ surface expression. Caki-1, KTC-26 and A498 cells were characterized by different basal integrin $\alpha$ and $\beta$ surface expression patterns (Fig. 4A). Caki-1 markedly expressed $\alpha 3$ and $\beta 1$, moderately expressed $\alpha 5$ and $\beta 3$, whereas $\alpha 1, \alpha 2, \alpha 4, \alpha 6$ and $\beta 4$ were only marginally detectable. KTC-26 strongly expressed $\alpha 3$ and $\beta 1$. The subtype members $\alpha 1, \alpha 2, \alpha 5, \alpha 6, \beta 3$ and $\beta 4$ were moderately expressed, and $\alpha 4$ was not detectable. The integrin expression profile of
A498 was similar to that of KTC-26, aside from $\alpha 4$, which was detectable for A498. We also noted that $\beta 4$ was present in KTC-26 cells but not in A498 cells.

Amygdalin application for twenty-four hours and for 2 weeks altered the integrin surface profile, which is specific to the cell type (Fig. 4B). We noted that $\alpha 5$ and $\alpha 6$ were significantly downregulated in all cell lines after 2 weeks of amygdalin exposure. $\beta 1$, which was strongly expressed in all three cell lines, was also significantly reduced following 2 weeks of amygdalin application. The high basal expression of the $\alpha 3$ receptor was reduced in Caki-1 and KTC-26 but not in A498 by amygdalin. Differences were also noted in relation to $\alpha 2$ and $\beta 3$, both of which were reduced in Caki-1 cells but elevated in KTC-26 and A498 cells after 2 weeks. Diminished expression levels of $\beta 4$ were found in Caki-1 and KTC-26 cells after amygdalin exposure.

Amygdalin influences the total cellular integrin content. Evaluation of the integrin protein content after $24 \mathrm{~h}$ of amygdalin exposure revealed significant upregulation of $\alpha 2$ and downregulation of $\alpha 3$ and p-FAK (Fig. 5). $\beta 1$ was significantly elevated in Caki-1 and KTC-26, whereas $\alpha 6$ was significantly decreased in Caki-1 and A498, and the total content of $\alpha 5$ was reduced in A498 cells after 24 h. In Caki-1 cells, $\alpha 4$ and $\beta 4$ increased and $\beta 3$ decreased. 

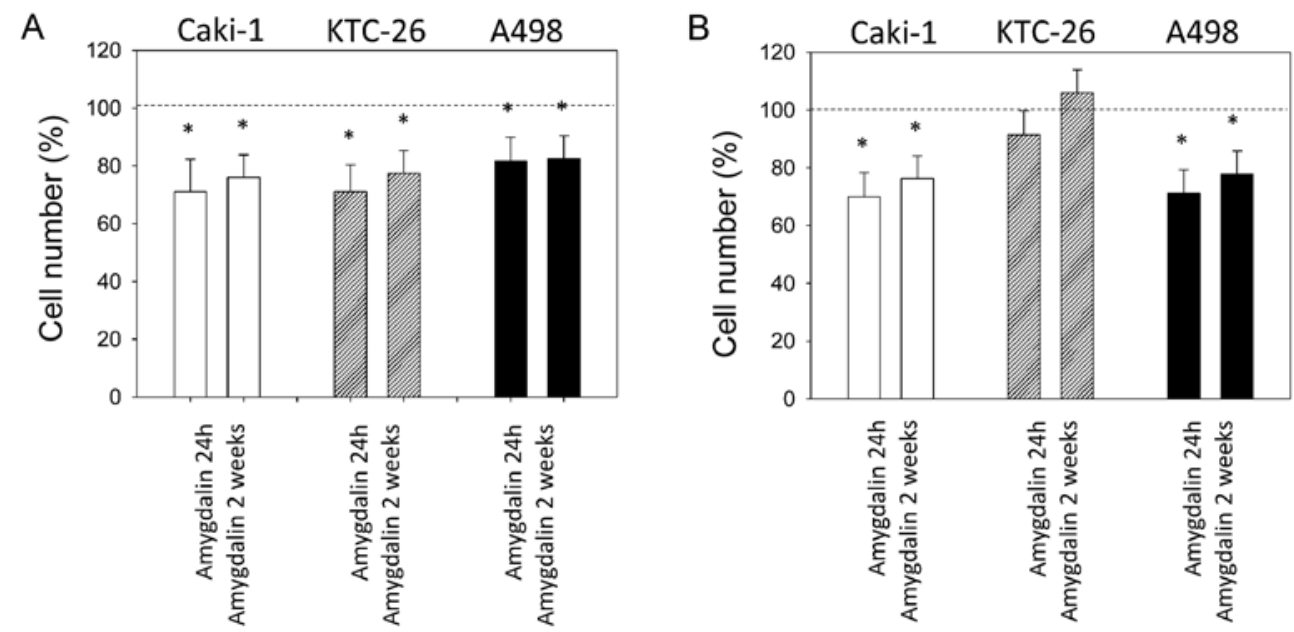

Figure 3. Effect of amygdalin on renal cell carcinoma (RCC) cells (A) chemotaxis and (B) invasion. RCC cells treated with amygdalin for $24 \mathrm{~h}$ or 2 weeks were seeded in the upper chamber with a chemoattractant in the well below. Cells migrating through the membrane after $20 \mathrm{~h}$ were counted. Controls received no amygdalin and were set to $100 \%$ (dotted line). ${ }^{*}$ Significant difference to controls. Bars indicate the the means \pm standard deviation (SD). $n=5$ experiments, $\mathrm{p} \leq 0.05$.
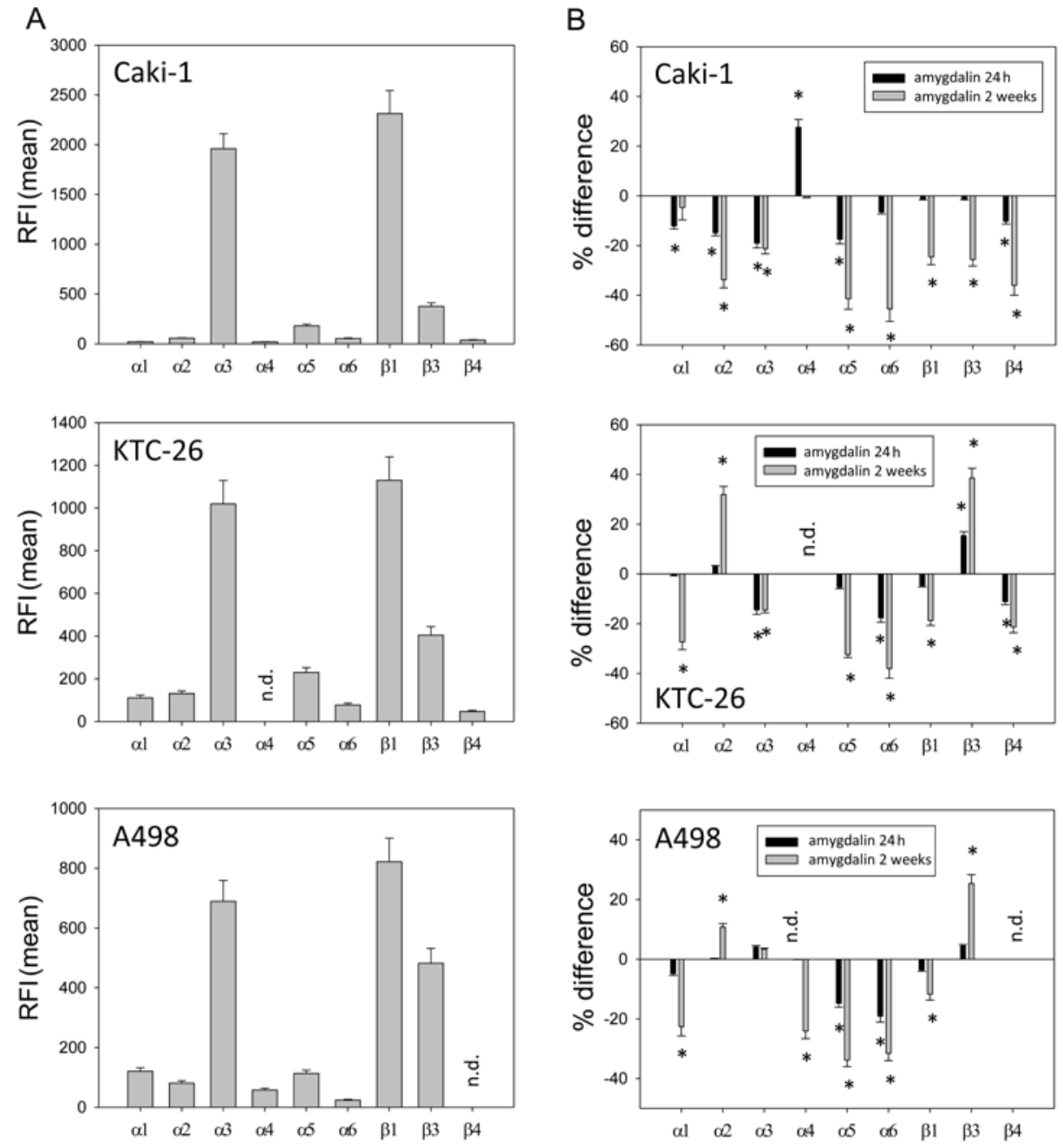

Figure 4. (A) Basal surface expression of renal cell carcinoma (RCC) integrin subtypes and (B) difference (\%) to untreated controls after $24 \mathrm{~h}$ or 2 weeks of amygdalin exposure. RFI, relative fluorescence intensity. n.d., not detectable. "Significant difference to controls. Bars indicate the means \pm standard deviation (SD), $\mathrm{p} \leq 0.05$.

After 2 weeks of exposure to amygdalin, integrin $\alpha 2$ significantly increased, even more so than after exposure for $24 \mathrm{~h}$. In A498 cells, $\alpha 3$ and $\beta 3$ increased after 2 weeks of amygdalin exposure. Reduced $\beta 1$ and p-FAK occurred in KTC-26 cells, and $\beta 4$ was downregulated in both Caki-1 and KTC-26 after 2 weeks of amygdalin exposure (Fig. 5). 
A

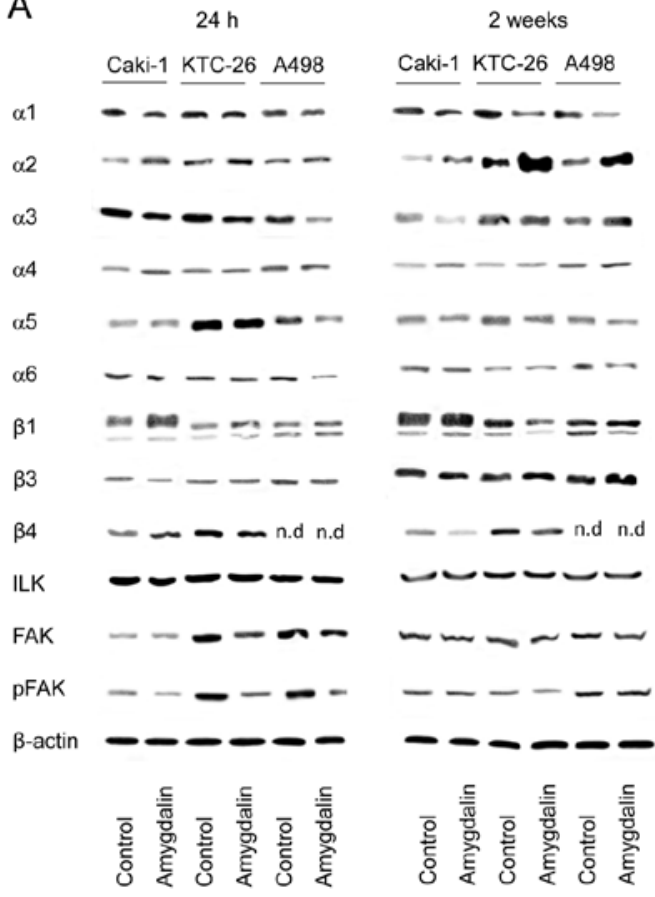

B
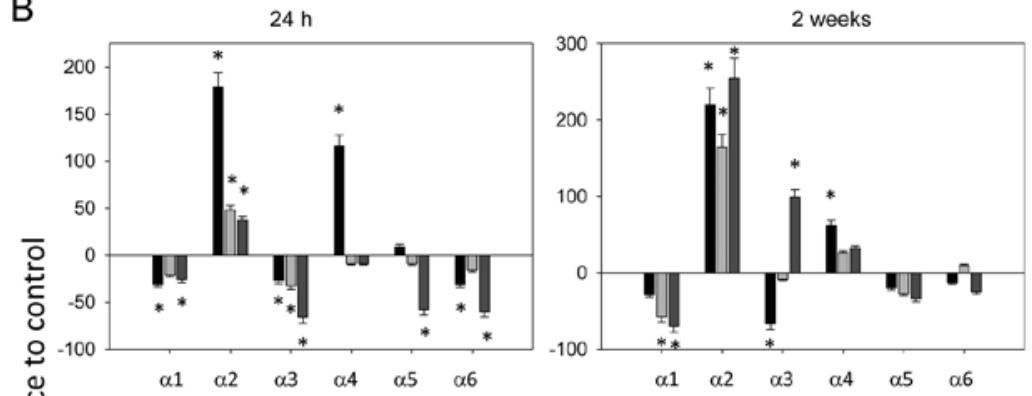

$\begin{array}{llllll}\alpha 1 & \alpha 2 & \alpha 3 & \alpha 4 & \alpha 5 & \alpha 6\end{array}$
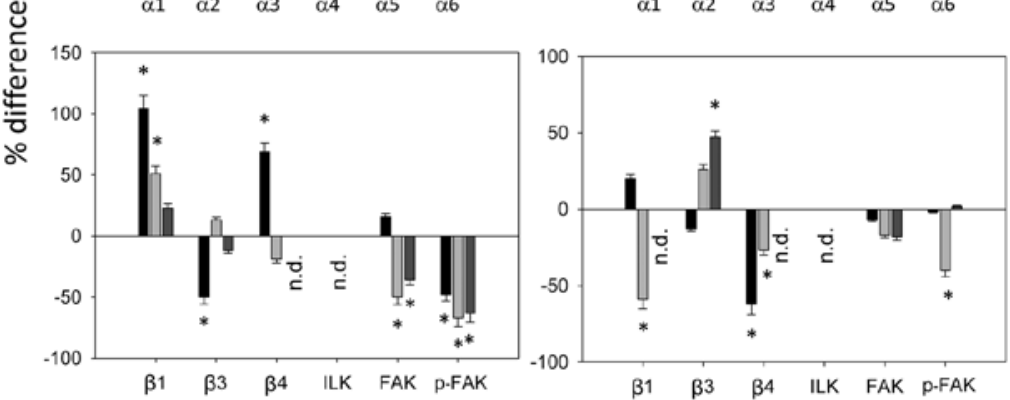

Figure 5. (A) Western blot analysis of total integrin content of Caki-1, KTC-26 and A498 cells exposed to amygdalin for 24 h or 2 weeks and untreated controls $\beta$-actin served as the internal control. (B) Pixel density analysis of the western blot analysis protein bands. The ratio of protein intensity/ $\beta$-actin intensity was calculated, and expressed as a percentage, related to controls set to $100 \%$ (0) after cells were exposed to amygdalin for 24 h or 2 weeks. One representative of three separate experiments is shown. n.d., not detectable. "Significant difference to controls, $\mathrm{p} \leq 0.05$.

A

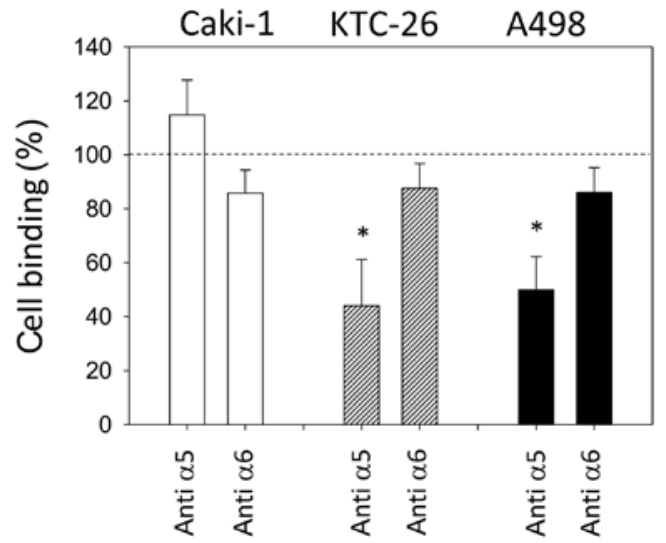

B

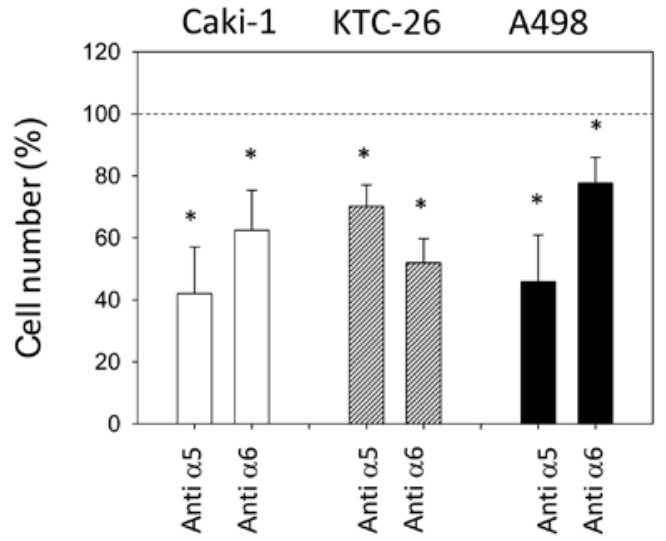

Figure 6. Influence of integrin $\alpha 5$ and $\alpha 6$ functional blocking on (A) cell adhesion to collagen and (B) chemotaxis. Unblocked cells served as controls (100\%, dotted line). The mean number of adherent or chemotactically active cells from five fields $\left(0.25 \mathrm{~mm}^{2}\right)$ was evaluated. Bars indicate the means \pm standard deviation (SD). *Significant difference to controls. $n=3$ experiments, $\mathrm{p} \leq 0.05$.
Blocking experiments. The integrin expression profiles of all three cell lines were modified by amygdalin. Since surface integrin $\alpha 5$ and integrin $\alpha 6$ were strongly reduced in all three cell lines following amygdalin application, these integrins were chosen for functional blocking studies, to investigate whether the reductions correlate with changes in tumor cell adhesion and migration. Blocking $\alpha 5$ led to the significant inhibition of KTC-26 and A498 cell adhesion to collagen (Fig. 6A). However, adhesion of Caki-1 cells to collagen was not significantly influenced. Blocking integrin $\alpha 5$ resulted in reduced chemotactic activity in all three cell lines (Fig. 6B). Blocking the $\alpha 6$ receptor did not significantly affect adhesion to collagen in any of the cell lines (Fig. 6A) but significantly decreased chemotaxis in all three cell lines (Fig. 6B).

\section{Discussion}

It has been noted that interaction between tumor cells and endothelium plays a crucial role in metastatic progression; adhesion of non-small cell lung cancer (NSCLC) cells to the vessel-wall endothelium has been associated with tumor cell transmigration, which leads to brain and lymph node metastases (7). A more aggressive, metastasizing cancer phenotype has also been associated with enhanced adhesion (7). In the present study, we demonstrated that amygdalin exposure led to significant inhibition of the binding interaction between RCC cells and a HUVEC monolayer, collagen and fibronectin. The chemotactic and invasive activity of RCC cells was thereby inhibited. Such inhibition is clinically relevant since transendothelial migration and motile spreading are critical steps in tumor dissemination and progression (3) and correlate with poor survival. Reducing 
migratory potential has been associated with successful tumor therapy and a less malignant tumor phenotype (8). Thus, we suggest that inhibiting RCC cell adhesion and motility by amygdalin reduces metastatic spread.

The adhesion- and migration-blocking effect of amygdalin is not restricted to RCC cells. Amygdalin has recently been demonstrated to also suppress the adhesive behavior of bladder cancer cells (5). Although amygdalin exerted a similar suppressive effect on the adhesion properties in bladder cancer and RCC cells, bladder cancer cell migration was affected differently by amygdalin. Chemotaxis was downregulated in two, but upregulated in one bladder cancer cell line following amygdalin exposure, indicating that the influence of amygdalin probably depends on the tumor entity. Thus, it is important to investigate the impact of amygdalin on different tumor entities.

The integrin family has been implicated in all steps of metastatic tumor progression $(4,7)$. Integrin $\alpha 5$ is upregulated in tumor cells of epithelial origin, and a positive correlation between integrin $\alpha 5$ expression and RCC cell adhesion has been established (9). In the present investigation, amygdalin administration significantly decreased surface integrin $\alpha 5$ in all three cell lines. Also, the total cellular content of integrin $\alpha 5$ time-dependently decreased in the presence of amygdalin. Blocking integrin $\alpha 5$ function caused significant inhibition of KTC-26 and A498 cell adhesion to collagen and a decrease in the chemotactic activity of all cell lines employed. Consistent with the present data, downregulation of integrin $\alpha 5$ has previously been associated with reduced adhesive and invasive behavior of several cancer cell types (10-12).

In the present study, we noted that the collagen adhesion of Caki-1 cells did not decrease after blocking integrin $\alpha 5$, in contrast to the amygdalin-induced decrease in A498 and KTC-26 cells. Such a difference in integrin function between tumor cell types has previously been observed. Blocking $\alpha 5$ integrin has been shown to inhibit cell-matrix interaction of the bladder cancer cells HCV29 and BC3726 but enhance binding of the bladder cancer cell lines T24 and Hu456 (equipped with a different integrin set) (13). Similarly, blocking $\beta 1$ integrin has been shown to inhibit UMUC-3 bladder cancer cell attachment to collagen, but has the opposite adhesive effect on TCCSUP cells, which are characterized by a different integrin expression profile (5). In the present investigation, amygdalin exposure caused surface $\beta 3$ integrin to increase in KTC-26 and A498 cells, but decrease in Caki-1 cells. Counter-regulation involving another integrin subtype, in this case $\beta 3$, may explain why blocking $\alpha 5$ failed to stop adhesion in Caki- 1 cells. Loss of integrin $\alpha 5$ caused chemotaxic reduction in all three cell lines. Therefore, we suggest that loss of surface integrin $\alpha 5$ is a mechanism by which amygdalin acts on RCC cell migration, and fine-tuning integrin performance depends on the specific integrin profile in the particular cell line.

Integrin $\alpha 6$ has been shown to facilitate epithelial cell migration and is correlated with progression risk, metastasis and death in clinical trials (14). Other studies have shown that integrin $\alpha 6$ promotes migration and invasion in colorectal cancer (15), and pancreatic (16) and breast (17) carcinomas. Integrin $\alpha 6$ has been noted to activate FAK (18) and FAK-related downstream signaling, which is relevant to controlling cell motility, survival and proliferation (4). In the present investigation, surface expression of integrin $\alpha 6$ was significantly reduced and FAK was deactivated by amygdalin in all three cell lines. Blocking surface integrin $\alpha 6$ demonstrated that $\alpha 6$ does not interfere with tumor cell adhesion but does regulate cell motility. Therefore, it is likely that reduction of $\alpha 6$ represents a mechanism by which amygdalin slows cell spreading.

Integrin $\beta 1$ has been shown to promote cell invasion in breast, lung, pancreatic and colorectal cancer, as well as glioma, melanoma (4) and neuroblastoma (19). In prostate cancer cells, upregulation of integrin $\beta 1$ has been shown to be accompanied by elevated motile behavior, whereas integrin $\beta 1$ blockade contributes to the downregulation of chemotaxis, migration and cell adhesion (10). Inhibition of integrin $\beta 1$ has been associated with reduced invasion and metastasis of ovarian cancer (12), and an integrin $\alpha 5 \beta 1$ peptide inhibitor has been shown to block breast cancer metastasis in vivo (20). In the present investigation, surface integrin $\beta 1$ was downregulated in all three tumor cell lines following 2 weeks of amygdalin exposure. Therefore, amygdalin may act on integrin $\beta 1$ to slow the motile spreading of RCC cells.

In vitro and in vivo investigations indicate that $\alpha 3$ is another integrin involved in the invasion of glioma, melanoma, hepatocellular and mammary carcinoma, and promotes lung metastasis of breast carcinoma cells (4). Blocking integrin $\alpha 3$ has resulted in adhesion inhibition of prostate cancer cells (21). In the present investigation, surface integrin $\alpha 3$ was reduced in Caki-1 and KTC-26, but not in A498 cells. This inhomogeneous reduction points to a cell line-specific effect induced by amygdalin.

Amygdalin application, besides modulating surface integrin expression, also changed total cellular integrin content. In the present study, total cellular integrin $\alpha 2$ expression was elevated in all three tumor cell lines following amydalin exposure. Previous studies have shown that decreased levels of integrin $\alpha 2$ in tumor cells potentially increase tumor cell dissemination, and re-expression of integrin $\alpha 2$ has been shown to reverse malignant properties of breast cancer cells (22). Hence, we suggest that the amygdalin-induced inhibition of tumor cell adhesion and migration observed here is associated with upregulation of integrin $\alpha 2$.

Total cellular $\alpha 3$ integrin was decreased $24 \mathrm{~h}$ after amygdalin application in all three cell lines. This is in line with the amygdalin-induced reduction in surface $\alpha 3$ in Caki-1 and KTC-26 cells. Since surface $\alpha 3$ was not modified in the A498 cells, it is possible that amygdalin in this cell line acts through the intracellular $\alpha 3$ signaling pathways. p-FAK, which was strongly diminished in A498 cells $24 \mathrm{~h}$ after amygdalin application, supports this hypothesis since the $\alpha 3-\mathrm{FAK}$ axis is involved in cancer initiation and progression (23). Lee et al have demonstrated that FAK is a critical mediator of tumorigenesis and metastasis, which in part depend on integrin $\alpha 3$ (24). Therefore, we suggest that knocking down both integrin $\alpha 3$ and FAK deactivates the motile machinery of A498 cells.

Total cellular integrin $\beta 1$ was elevated in Caki-1 cells after amygdalin application, while surface expression was diminished. This type of shift is not uncommon and points to receptor translocation from the surface to the intracellular compartment. Although the relevance of this process is not fully understood, integrin $\beta 1$ trafficking to the plasma membrane has been shown to increase the metastatic potential of RCC cells, whereas stopping $\beta 1$ recycling by maintaining 
a high cyctoplasmic and a low plasma membrane content decreases metastasis (25). Elevation of intracellular $\beta 1$ has been shown to be linked with FAK deactivation (25), which correlates with the present findings.

The effects of amygdalin on integrin subtype expression depended on the cell line, upon whether the application time was acute $(24 \mathrm{~h})$ or chronic ( 2 weeks) and whether the integrin location was the cell surface or in the cytoplasm. The molecular mode of action of amygdalin in regard to integrin subtype expression, therefore, is not homogeneous and may influence both integrin-triggered mechanical cell-to-cell coupling and integrin-controlled biochemical pathway activation.

In the three investigated RCC cell lines, amygdalin application significantly reduced invasive and motile behavior. However, 2 weeks of amygdalin did not reduce the invasive capacity of KTC-26 cells. The reduction was predominantly associated with a decrease in surface $\alpha 5$ and $\alpha 6$ integrins. This mechanism, however, should not be generalized. Although amygdalin has been shown to inhibit adhesion and migration in bladder cancer cells as well, the integrins are altered differently. The $\alpha 5$ and $\alpha 6$ integrins seem to be an important target of amygdalin in RCC cells, whereas modulation of $\beta 1$ or $\beta 4$ integrins was most apparent in bladder cancer cells (5). Further in vitro investigations have been initiated to evaluate whether amygdalin also influences RCC cell growth, as has been observed for bladder cancer cells (6).

In conclusion, exposing RCC cells to amygdalin inhibits metastatic spread and is associated with downregulation of $\alpha 5$ and $\alpha 6$ integrins. Therefore, amygdalin exerts antitumor activity in vitro in RCC. This in vitro activity must be evaluated in an animal model.

\section{Acknowledgements}

The present study was supported by the 'Brigitta und Norbert Muth Stiftung' and the 'Freunde und Förderer der Goethe-Universität Frankfurt'.

\section{References}

1. Moertel CG, Fleming TR, Rubin J, Kvols LK, Sarna G, Koch R Currie VE, Young CW, Jones SE and Davignon JP: A clinical trial of amygdalin (laetrile) in the treatment of human cancer. N Engl J Med 306: 201-206, 1982.

2. Newell GR and Ellison NM: Ethics and designs: laetrile trials as an example. Cancer Treat Rep 64: 363-365, 1980.

3. van Zijl F, Krupitza G and Mikulits W: Initial steps of metastasis: cell invasion and endothelial transmigration. Mutat Res 728: 23-34, 2011.

4. Sun CC, Qu XJ and Gao ZH: Integrins: players in cancer progression and targets in cancer therapy. Anticancer Drugs 25 : $1107-1121,2014$

5. Makarević J, Rutz J, Juengel E, Kaulfuss S, Tsaur I, Nelson K, Pfitzenmaier J, Haferkamp A and Blaheta RA: Amygdalin influences bladder cancer cell adhesion and invasion in vitro. PLoS One 9: e110244, 2014.

6. Makarević J, Rutz J, Juengel E, Kaulfuss S, Reiter M, Tsaur I, Bartsch G, Haferkamp A and Blaheta RA: Amygdalin blocks bladder cancer cell growth in vitro by diminishing cyclin A and cdk2. PLoS One 9: e105590, 2014

7. Salvo E, Garasa S, Dotor J, Morales X, Peláez R, Altevogt P and Rouzaut A: Combined targeting of TGF- $\beta 1$ and integrin $\beta 3$ impairs lymph node metastasis in a mouse model of non-small-cell lung cancer. Mol Cancer 13: 112, 2014.
8. White NM, Masui O, Newsted D, Scorilas A, Romaschin AD, Bjarnason GA, Siu KW and Yousef GM: Galectin-1 has potential prognostic significance and is implicated in clear cell renal cell carcinoma progression through the HIF/mTOR signaling axis. $\mathrm{Br}$ J Cancer 110: 1250-1259, 2014.

9. Juengel E, Makarević J, Reiter M, Mani J, Tsaur I, Bartsch G, Haferkamp A and Blaheta RA: Resistance to the mTOR inhibitor temsirolimus alters adhesion and migration behavior of renal cell carcinoma cells through an integrin $\alpha 5$ - and integrin 33-dependent mechanism. Neoplasia 16: 291-300, 2014.

10. Tsaur I, Makarević J, Juengel E, Gasser M, Waaga-Gasser AM, Kurosch M, Reiter M, Wedel S, Bartsch G, Haferkamp A, et al: Resistance to the mTOR-inhibitor RAD001 elevates integrin $\alpha 2$ - and $\beta 1$-triggered motility, migration and invasion of prostate cancer cells. Br J Cancer 107: 847-855, 2012.

11. Imanishi Y, Hu B, Jarzynka MJ, Guo P, Elishaev E, Bar-Joseph I and Cheng SY: Angiopoietin-2 stimulates breast cancer metastasis through the alpha(5)beta(1) integrin-mediated pathway. Cancer Res 67: 4254-4263, 2007

12. Mitra AK, Sawada K, Tiwari P, Mui K, Gwin K and Lengyel E: Ligand-independent activation of c-Met by fibronectin and $\alpha(5)$ $\beta(1)$-integrin regulates ovarian cancer invasion and metastasis. Oncogene 30: 1566-1576, 2011.

13. Lityńska A, Przybyło M, Pocheć E and Laidler P: Adhesion properties of human bladder cell lines with extracellular matrix components: the role of integrins and glycosylation. Acta Biochim Pol 49: 643-650, 2002.

14. Ricci E, Mattei E, Dumontet C, Eaton CL, Hamdy F, van der Pluije G, Cecchini M, Thalmann G, Clezardin P and Colombel M: Increased expression of putative cancer stem cell markers in the bone marrow of prostate cancer patients is associated with bone metastasis progression. Prostate 73: 1738-1746, 2013.

15. Chao C, Lotz MM, Clarke AC and Mercurio AM: A function for the integrin alpha6beta4 in the invasive properties of colorectal carcinoma cells. Cancer Res 56: 4811-4819, 1996.

16. Cruz-Monserrate Z and O'Connor KL: Integrin alpha 6 beta 4 promotes migration, invasion through Tiam1 upregulation, and subsequent Rac activation. Neoplasia 10: 408-417, 2008.

17. Kim TH, Kim HI, Soung YH, Shaw LA and Chung J: Integrin (alpha6beta4) signals through Src to increase expression of S100A4, a metastasis-promoting factor: implications for cancer cell invasion. Mol Cancer Res 7: 1605-1612, 2009.

18. Yang XH,Flores LM, Li Q,Zhou P, Xu F, Krop IE and Hemler ME: Disruption of laminin-integrin-CD151-focal adhesion kinase axis sensitizes breast cancer cells to ErbB2 antagonists. Cancer Res 70: 2256-2263, 2010.

19. Lee S, Qiao J, Paul P and Chung DH: Integrin $\beta 1$ is critical for gastrin-releasing peptide receptor-mediated neuroblastoma cell migration and invasion. Surgery 154: 369-375, 2013.

20. Khalili P, Arakelian A, Chen G, Plunkett ML, Beck I, Parry GC, Doñate F, Shaw DE, Mazar AP and Rabbani SA: A non-RGD-based integrin binding peptide (ATN-161) blocks breast cancer growth and metastasis in vivo. Mol Cancer Ther 5: 2271-2280, 2006

21. Tsaur I, Rutz J, Makarević J, Juengel E, Gust KM, Borgmann H, Schilling D, Nelson K, Haferkamp A, Bartsch G and Blaheta RA: CCL2 promotes integrin-mediated adhesion of prostate cancer cells in vitro. World J Urol 33: 1051-1056, 2015.

22. Zutter MM, Santoro SA, Staatz WD and Tsung YL: Re-expression of the alpha 2 beta 1 integrin abrogates the malignant phenotype of breast carcinoma cells. Proc Natl Acad Sci USA 92: 7411-7415, 1995.

23. Cagnet S, Faraldo MM, Kreft M, Sonnenberg A, Raymond K and Glukhova MA: Signaling events mediated by $\alpha 3 \beta 1$ integrin are essential for mammary tumorigenesis. Oncogene 33: 4286-4295, 2014.

24. Lee S, Qiao J, Paul P, O'Connor KL, Evers MB and Chung DH: FAK is a critical regulator of neuroblastoma liver metastasis. Oncotarget 3: 1576-1587, 2012.

25. Tringali C, Lupo B, Silvestri I, Papini N, Anastasia L, Tettamanti G and Venerando B: The plasma membrane sialidase NEU3 regulates the malignancy of renal carcinoma cells by controlling $\beta 1$ integrin internalization and recycling. J Biol Chem 287: 42835-42845, 2012. 